INSTITUT NATIONAL DE RECHERCHE EN INFORMATIQUE ET EN AUTOMATIQUE

\title{
Geometrically Exact Dynamic Splines
}

Adrien Theetten - Laurent Grisoni - Claude Andriot — Brian Barsky

\section{$\mathbf{N}^{\circ}$ ????}

Novembre 2006

Thème NUM

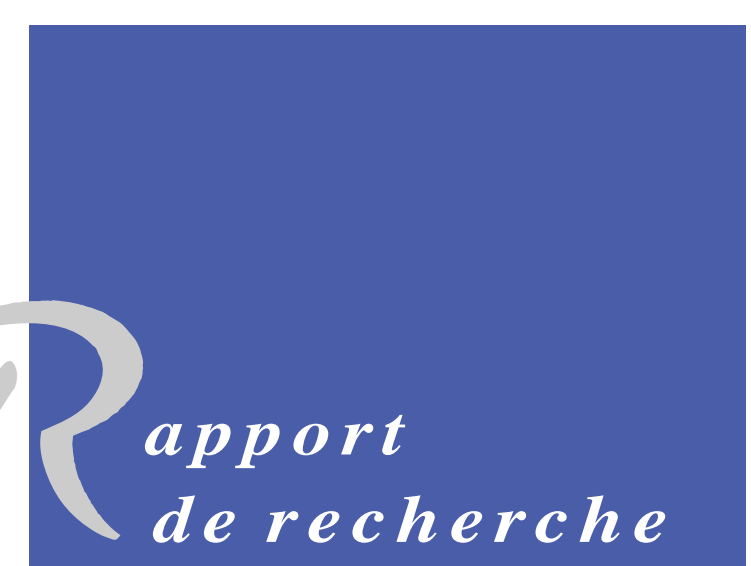





\title{
RINRIA
}

\section{Geometrically Exact Dynamic Splines}

\author{
Adrien Theetten ${ }^{*}{ }^{\dagger}$, Laurent Grisoni ${ }^{\ddagger} \dagger$, Claude Andriot ${ }^{\S}$, Brian Barsky $₫$ \\ Thème NUM — Systèmes numériques \\ Projet Alcove \\ Rapport de recherche n ???? — Novembre 2006 - 30 pages
}

\begin{abstract}
In this paper, we propose a complete model handling physical simulation of deformable 1D objects. We formulate continuous expressions for stretching, bending and twisting energies. These expressions are mechanically rigorous and geometrically exact. Both elastic and plastic deformations are handled to simulate a wide range of materials. We validate the proposed model on several classical test configurations. The use of geometrical exact energies with dynamic splines provides very accurate results as well as interactive simulation time, which shows the suitability of the proposed model for constrained CAD applications. We illustrate the application potential of the proposed model by describing a virtual system for cable positioning, that can be used to test compatibility between planned fixing clip positions, and mechanical cable properties.
\end{abstract}

Key-words: spline, beam theory, elasticity, plasticity, Lagrange equations.

\footnotetext{
* adrien.theetten@lifl.fr

$\dagger$ LIFL, IRCICA/INRIA Futurs, USTL France

¥ laurent.grisoni@lifl.fr

$\S$ claude.andriot@cea.fr, CEA, Fontenay-aux-Roses, France

ฯ barsky@cs.berkeley.edu, University of California, Berkeley, USA
} 


\section{Geometrically Exact Dynamic Splines}

Résumé : Nous proposons ici un modèle physique complet d'objets 1D déformables. Nous formulons les expressions continues des énergies d'élongation, de flexion et de torsion. Celles-ci sont mcaniquement rigoureuses et géométriquement exactes. Les déformations élastiques et plastiques sont prises en compte afin de simuler un large éventail de matériaux. Nous validons le modèle proposé sur plusieurs expériences classiques. L'utilisation conjointe d'énergies géométriquement exactes et de splines dynamiques permet aussi bien des résultats très précis que des simulations en temps interactif, ce qui montre l'adéquation du modèle proposé avec la conception assistée par ordinateur sous contraintes. Nous illustrons le potentiel du modèle proposé par un système de pose virtuelle de câbles, qui peut être utilisé pour tester la compatibilité entre la position de clips de fixation et les propriétés mécaniques d'un câble.

Mots-clés : spline, théorie des poutres, elasticité, plasticité, équations de Lagrange 


\section{Introduction}

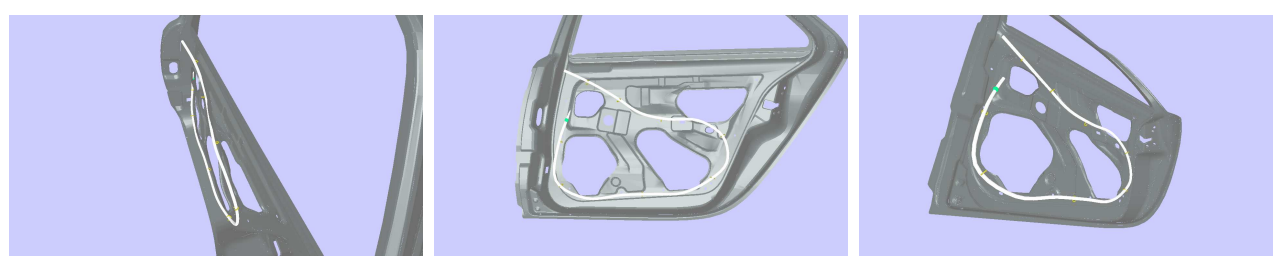

Figure 1: Virtual cable positioning on a car door.

One-dimensional flexible models are a CAD key element in number of practical situations. Cables of largely varying mechanical properties are nowadays used in industry. In fields such as car and plane design, virtual prototyping is used to improve quality and to reduce development costs. As a matter of fact, virtual prototyping includes more and more assembly simulations: it allows early detection of potential problems, and also permits to study ease of assembly. This implies to be able to accurately represent geometry, but also mechanical behavior of involved parts. Among the many objects to be simulated, flexible one-dimensional objects are of significant importance. They are involved in vehicle engineering (e.g. electrical cable laying within the car structure [[Fl]), but also in fields such as architecture (e.g. stiff electrical cable positioning within virtual buildings), or even in medical simulation (surgical thread simulation is currently an active research question in the medical simulation community [LCDN06]). For most unconstrained Computer Aided Design applications, splines are probably the most classical tool for 1D objects. As a matter of fact, NURBS have become an industry-standard representation for 1D objects. Dynamic splines have been introduced by Qin and Terzopoulos [QT96]. They combine physics-based constraining equations to spline geometry, in order to improve the design process. In this article, we propose an approach that extends the mechanical accuracy of previously proposed approaches. We propose, anywhere possible, geometrically exact formal expressions that, along with spline analytical expressions, provide a powerful, real-time model. We call this model Geometrically Exact Dynamic Splines, or GEDS for short.

In this paper we propose a spline-based model for real-time, mechanically accurate, simulation of one-dimensional objects. Our model can handle both reversible (elastic) and irreversible (plastic) deformations. The proposed formalism and energy expressions model stretching, bending and twisting loads; at the very limit of material constraint, we show how our model can be used to detect break points. We also show that interactive rate is provided for a wide range of configurations. Finally, we describe a practical application of our model, that permits to virtually validate electrical cable positioning and clipping along a path on a car door. Our specific scientific contributions are the following:

An improvement of dynamic spline state-of-the-art, namely a formulation of stretching, twisting and bending deformations in large rotations (or large displacements), through 
geometrically exact energy expressions. Such terms allow accurate results, while still running in interactive time. The proposed method is fully compatible with Lagrangian multipliers;

The handle of twisting with an extended spline formulation, by decomposition of twisting in a geometrical part and a roll part. Such a separation ensures numerical stability for twisting energy evaluation. In addition to that, solving of the proposed mechanical model does not require a local frame, which makes it all the more accurate (frames are classically stabilized along a 1D curve using non-mechanical methods, see section $8)$;

An easy and efficient inclusion of plasticity within the Lagrange spline model.

The remainder of the paper is organized as follows: next section describes related work. In section 3, we provide a short summary of elasticity and plasticity theory, that constitutes the core mechanical knowledge for understanding the remainder of the article. Then we define in section 4 the formalism we use to describe geometrical model configurations. In section 5 , we propose a method (including elegant formalism) handling elastic deformations of the model, in a geometrically exact manner. In section 6 , we show how elastic deformation simulation can be combined with plastic behavior detection and simulation. For the sake of completeness, we provide in section 7 the (classical) tools we use to handle worlds interaction with the $1 \mathrm{D}$ model. Section 8 provides some comments about twisting handling in the proposed deformation model, which is one of the very crucial points in the method. Finally, section 9 describes tests and practical results:first, for ease of understanding, we provide a complete overview of the animation algorithm, which links the equations all together. Second, we compare numerical results of our model to several classical reference configurations. Third, we describe an advanced practical application of the model:a virtual system for electrical cable position testing on a car door (see visual example of Fig. 1).

\section{Previous Works}

Constraints solver has now become a standard part of most CAD modelers, and is still a very active research field [MFLS06]; the range of applications of such techniques is potentially very large (e.g see $\left.\left[\mathrm{BKV}^{+} 02\right]\right)$. Constraint solving most often relates to finding a compatible solution between user modeling requirements, and pre-imposed geometric constraints. Variational modeling[WW92] minimizes the global energy of a constrained geometric deformable object, and can be seen as an introduction of physical behavior into constraint solving. Physics-based modeling is more and more involved into the field of constrained geometrical design: it permits to extend constraints to the intrinsic mechanical properties of the modeled object.

Study of one-dimensional deformable objects has been a recurrent problem in computer graphics for about 20 years [TPBF87]. Many existing results, from most computationally efficient to most numerically accurate, try to catch complexity of one-dimensional deformable 
models. Simple models, like particles, networks of mass-springs[JL04] or rigid articulated bodies[RGL05], provide solutions on arbitrary linear discretization. Most of these models provide fast and interactive simulations, but are difficult to precisely tune for some given material, as their parameters do not directly relate to the coefficients provided by mechanic. These shortcomings have been addressed by developing more accurate models that underly physics. Inspired by classical numerical simulation methods, finite element and boundary element methods (see [JP99], $\left[\mathrm{MDM}^{+} 02\right]$ ) are known to yield accurate numerical results for fine discretization of the studied object; such an accuracy is difficult to combine with reasonable computation time in practice.

In robotics community, several recent works use Cosserat theory. Cosserat medium was first described in 1909 by Cosserat brothers[CC09]. This medium is described by a set of oriented micro-solids. Pai [Pai02] first introduced Cosserat's rod theory in computer graphics to model cantilever objects. In this work the animation step is done in two passes: the first one calculates the forces and torques iteratively along the rod discretization, the second one evaluates the geometrical configuration in backward iterations. Wakamatsu [WH04] achieves a very accurate static solution of a cable simulation by considering it as a succession of oriented frames and by minimizing its potential energy; this approach is mechanically accurate, but demands very high computation cost. Bertails \& al $\left[\mathrm{BAC}^{+} 06\right]$ define Super-Helices for simulating dynamics of human hair strands. A Lagrangian formulation of inextensible Kirchhoff rods is used. This method is fast to compute and visually realistic for low resolution rods; the quadratic complexity of the algorithm is still a key problem for real-time simulation of high complexity rods. The main drawback of these three methods is that it is difficult to combine such models to constrains. Moreover, these methods need at least a reference point for calculation, that might not always be available in practical cases (e.g. modeling rest state position of a deformable cable within a recipient shape). Gregoire [GS06] very recently proposed a mass-spring model based on Cosserat theory, that resembles realistic and interactive twisting and bending deformations. This model uses consistent mechanic, and is based on simple, continuous, energy terms that provide efficient computation time; it is anyhow unclear in which measure this model is numerically accurate, if compared to real-life objects with known material properties.

Spline-based techniques are still quite isolated within physical animation literature. Terzopoulos \& al initiated deformable models in Computer Graphics, including physics-based curves [TPBF87], using a lagrangian form of newton equation. The model of Qin and Terzopoulos [QT96], Dynamic Non-Uniform Rational B-Splines (DNURBS), first combined spline representation with physics laws. Nocent and Rémion [NR01] define the Dynamic Material Splines (DMS), a full Lagrange-based simulation framework for splines. They consider spline control points as the degrees of freedom of the underlying continuous object. Continuous stretching energy is defined. Lagrange multipliers are used to constrain point position and tangent orientation. Lenoir [LMGC04] propose a curvature energy formulation for DMS that is not geometrically exact, but that provides real-time manipulations, as well as adaptive simulations[LGMC05]. We do not address in the present article the simulation of 
fracture: Lenoir \& al [LGMC05] provide an elegant manner to compute topological changes, by combining lagrangian mechanics with B-Splines knot-refinement properties.

None of the previously mentioned works deals with irreversible deformations. Onedimensional objects like electrical wires, telephone cables, suturing threads remain partially deformed after relaxation. Few papers deal with plasticity. Terzopoulos \& al [TF88] first introduced non-elastic behavior in Computer Graphics community. They proposed physicallybased models to simulate viscoelasticity, plasticity and fracture effects, for the purposes of animation, in the case of volumic objects. [WSG05] describes a point-based method for animation, that can handle fracture on shells. O'Brien \& al[OBH02] propose a method for realistic ductile fracture animation in common solid materials, such as plastics and metals. In the present work we use a similar decomposition of strains to handle plasticity.

\section{Mechanics Background}

This section provides a very short overview of the mechanical background, necessary for understanding the extension of Dynamic spline we propose, in the next section.

When a force is applied on a deformable object, object geometry is extended or compressed, and local topology may even change (i.e. material might break) if the force is large enough. Although both load and extension/compression are primary quantities, material scientists tend to use two derived quantities, stress $\sigma$ and strain $\varepsilon$, to analyze materials under load. Stress is the force per unit area and strain is the extension/compression per unit length. In the general case, both $\sigma$ and $\varepsilon$ are manipulated using tensors. However, in most practical cases, the relationship between the stress applied to a material and the resulting strain is described in mechanics by a simple relation separately in each direction: a curve can be produced that is characteristic of the material. Figure 2 is a typical representation of a stress-strain curve.

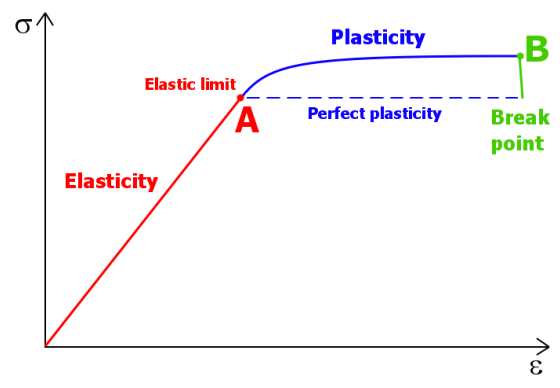

Figure 2: Schematic representation of a typical stress-strain curve. Elasticity is usually linear, whereas plasticity is assumed to be perfect. 
Table 1: Physical parameters for several materials[How01]

\begin{tabular}{|l|c|c|c|}
\hline Material & $E(G P a)$ & $\nu$ & $\mu\left(M g / \mathrm{m}^{3}\right)$ \\
\hline Nylon & 2.7 & 0.33 & 1.1 \\
Alluminium alloys & 71.7 & 0.34 & 2.8 \\
Copper & 120.7 & 0.35 & 8.9 \\
Steel, carbon & 206.8 & 0.28 & 7.8 \\
Steel, stainless & 189.6 & 0.28 & 7.8 \\
\hline
\end{tabular}

The behavior of the material fully depends on strain magnitude: elastic (i.e. reversible) deformations occur for small strains, plasticity (i.e. non-reversible) for more significant strain magnitudes. When strain intensity reaches the fracture point, geometry local topology changes, and continuous mechanics can no longer be applied.

\subsection{Elasticity}

When strain magnitude is relatively small (assumption of small strains in mechanics), it can be considered as directly proportional to stress (Hooke law). The elasticity domain only contains reversible deformations: when stress is relaxed, the material returns to its rest state. A nonlinear elastic region sometime needs to be studied, depending on the material: the mechanical model is then said to be defined for large strains. In practice, a wide range material available in real-life one-dimensional objects do not need such non-linear methods for good simulation accuracy. A a result, we only consider in this paper small strains (we show in section 9.2 that this approximation anyhow allows to reach good mechanical accuracy).

Several parameters characterize an elastic material. The longitudinal rate and the transversal rate between a stress and a related strain (i.e. the strain in the direction and the strain orthogonal to the stress, respectively) are given by Young modulus E (longitudinal elasticity modulus) and shear modulus $G$ (transversal elasticity modulus), respectively.These two moduli are interrelated by a formula incorporating Poisson's ratio $\nu$, which links longitudinal and transversal rates:

$$
G=\frac{E}{2(1+\nu)}
$$

The units of the two moduli are Pascals $\left(1 P a=1 N \cdot m^{-2}\right)$ whereas Poisson's ratio is dimensionless; specific values for several materials are provided in table 1 . These parameters completely identify a given material.

\subsection{Plasticity and fracture}

If the stress in a material exceeds the elastic limit A, also called yield point, for the material, then the stress is no longer linearly proportional to strain, and the deformations become nonreversible; when the stress is relaxed, the material remains partially deformed. After a plastic 
deformation, the strain-stress curve and the elastic region is translated along the strain axis. In the case of perfect plasticity, measured stress is independent of the applied strain: external work above the threshold value is fully turned into material, non-reversible, deformations. Under growing strain, when material strain limit is reached, a break (potentially fracture if applied strain is more important) occurs.

These material properties are handled by simulation using equations that define the dynamic relation between the geometry of the object (i.e. its deformations) and the applied forces.

\subsection{Energy balance}

The principle of least action, first formulated by de Maupertuis in 1747, is that nature always finds the most efficient path from one configuration to another. The Lagrange equations are deduced from this principle. They involve the kinetic energy $T$ and the potential energy $U$ of the system. The kinetic energy is the energy of motion whereas the potential energy is the stored energy of position possessed by an object. $F$ is the sum of external forces. Assuming the mass distribution to be homogeneously distributed between the $n$ degrees of freedom $\mathbf{q}_{\mathbf{i}}$ of the object, the Lagrange equation, that can be used for movement resolving is formulated as follows:

$$
\forall i \in\{1, \ldots, n\}, \frac{d}{d t}\left(\frac{\partial T}{\partial \dot{\mathbf{q}}_{\mathbf{i}}}\right)=\mathbf{F}_{i}-\frac{\partial U}{\partial \mathbf{q}_{\mathbf{i}}}
$$

In the case of Dynamic Splines, control points are used as the degrees of freedom if the object.

\section{GEDS definition}

\subsection{Beam geometry definition}

Beam theory is the study of one-dimensional objects in mechanics. Consider a cross-section of diameter $D$ and area $S$, as shown in Figure 3. The neutral fiber or neutral axis, denoted $f$, is the oriented curve of length $L$ that passes through the center of every cross-section. The volume defined by these cross-sections is a beam.

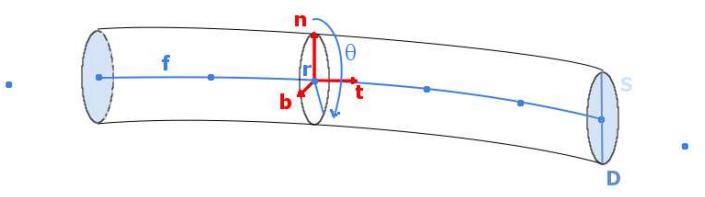

Figure 3: Scheme of a beam with its geometrical parameters and local frame. 


\subsection{Spline formulation}

The beam configuration is entirely described by two fields: a position field $\mathbf{r}=(x, y, z)$, which determinates neutral fiber $f$ position, and a rotation field $\theta$, which provides the roll of the cross-section. We propose to combine these two fields in a unique field described by a set of polynomial spline curves: $\mathbf{q}=(\mathbf{r}, \theta)=(x, y, z, \theta)$. Each resulting spline is given by

$$
\mathbf{q}(u)=\sum_{i=1}^{n} b_{i}(u) \mathbf{q}_{\mathbf{i}}
$$

where $b_{i}$ are the $i^{\text {th }}$ spline basis functions of the control points $\mathbf{q}_{\mathbf{i}}$, and $u$ is between 0 and $\ell$, the length of the neutral fiber. The $j^{\text {th }}$ derivative of $q$ with respect to $u$ is given by:

$$
\mathbf{q}^{(\mathbf{j})}(u)=\sum_{i=1}^{n} b_{i}{ }^{(j)}(u) \mathbf{q}_{\mathbf{i}} .
$$

Arc length is denoted by $s$. The derivative of control point $\mathbf{q}$, position $\mathbf{r}$ and roll $\theta$ with respect to $u$ are denoted by $\mathbf{q}^{\prime}, \mathbf{r}^{\prime}$ and $\theta^{\prime}$ respectively. The displacement elements $d s$ and $d u$ are interrelated by $d s=\left\|r^{\prime}\right\| d u$.

Since control points completely define the position of the spline and the orientation of the cross-sections, they can be considered as the degrees of freedom of the system and used in the Lagrange equations (2).

Although any kind of spline can be used, we have selected the cubic interpolatory Catmull-Rom spline for its efficiency and its ease of computation and the non-uniform rational B-spline (NURBS) to exploit adaptive subdivision.

Once we have described our one-dimensional object geometrically with splines, we may now use mechanics to make it evolve in space. In the following subsection, we define the physical part of the spline, using linear elastic but geometrically exact deformations.

\section{GEDS in the elastic domain}

To obtain the motion of control points with the Lagrange equations, deformation energies must be first formulated from physical parameters, and then differentiated with respect to the degrees of freedom. In this section, we propose a unified formulation to describe the deformations of a one dimensional object and the exact calculation of the corresponding forces.

\subsection{Constitutive laws and strain energies}

Every action on a beam can be modeled by forces and torques on the neutral fiber $f$; we express the force and the torque in the local frame instead of using stresses. They are here proportional to stresses and moreover easier to manipulate.

We elucidate here some terminology and notation, as shown in figure 4. 


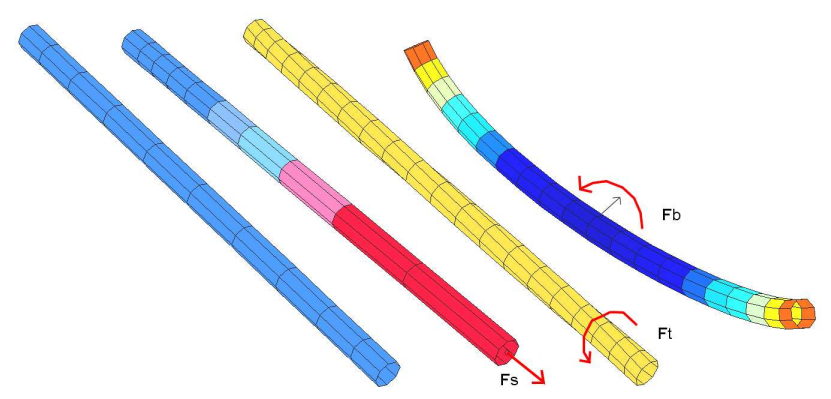

Figure 4: From left to right: rest state, stretching, twisting and bending on a beam.

The normal force to the cross-section, which results in stretching, is denoted by $\mathcal{F}_{S}$. The cross-section may rotate around the neutral fiber, due to the torsional torque, denoted by $\mathcal{F}_{T}$. The bending torque, denoted by $\mathcal{F}_{B}$, corresponds to the oriented curvature of the neutral fiber $f$. The Kirchhoff assumption presumes that cross-sections are stiff; only the neutral axis is distorted : we neglect shearing, force in the plane of the cross-section. This yields the Rayleigh model.

Let define the vector $\mathcal{F}$ as the data of $\mathcal{F}_{S}, \mathcal{F}_{T}$ and $\mathcal{F}_{B}$ :

$$
\mathcal{F}=\left(\begin{array}{c}
\mathcal{F}_{S} \\
\mathcal{F}_{T} \\
\mathcal{F}_{B}
\end{array}\right)
$$

$\mathcal{F}$ is related to the strain $\epsilon$. The rest strain is denoted $\epsilon^{0}$.

The elastical relationship between $\epsilon$ and $\mathcal{F}$ is well described in beam theory by Courbon [Cou80]. Considering linear elasticity facilitates the calculation of strain energies of a beam, as strains are directly proportional to stresses:we use the small strain assumption, which is accurate if the radius of curvature is large relative to the radius of the cross-section (usually about 5 times).

From these considerations, we provide the following result, which derives from Hooke's law:

$$
\mathcal{F}=\mathbb{H}\left(\epsilon-\epsilon^{0}\right)=\left(\begin{array}{ccc}
E S & 0 & 0 \\
0 & G I_{o} & 0 \\
0 & 0 & E I_{s}
\end{array}\right)\left(\epsilon-\epsilon^{0}\right)
$$

where $I_{o}$ is the polar momentum of inertia whereas $I_{s}$ is the cross-section momentum of inertia; $E S, G I_{o}$ and $E I_{s}$ are the stretching, the twisting and the bending rigidities respectively (see $[\mathrm{Cou} 80])$. We call $\mathbb{H}$ the Hooke matrix. 
Assuming cross-section is circular and its diameter constant, we can obtain the following expression of $\mathbb{H}$ :

$$
\mathbb{H}=\frac{D^{2} \pi}{4}\left(\begin{array}{ccc}
E & 0 & 0 \\
0 & \frac{G D^{2}}{8} & 0 \\
0 & 0 & \frac{E D^{2}}{16}
\end{array}\right)
$$

Strain energy $U$ is formulated by the following integration along the beam:

$$
U=\frac{1}{2} \int_{0}^{L}\left(\epsilon-\epsilon^{0}\right)^{t} \mathcal{F} d s
$$

Using expression 6 of $\mathcal{F}$, we get:

$$
U=\frac{1}{2} \int_{0}^{L}\left(\epsilon-\epsilon^{0}\right)^{t} \mathbb{H}\left(\epsilon-\epsilon^{0}\right) d s
$$

We now have all the background in mechanics necessary to determine the motion of our one-dimensional object by solving the Lagrange equations 2 . In the two next subsections, we will study the two terms of the equations so as to obtain a numerical solution.

\subsection{Handling twisting in Dynamic Splines}

Since the one-dimensional object is specified by position and rotation, its kinetic energy comprises translation energy and rotational energy. Translation energy corresponds to the displacement of control points and rotation energy is due to the motion of cross-sections around the neutral axis. We define the inertia matrix, denoted $\mathbb{J}$, which is the same everywhere along the spline, since diameter is constant:

$$
\mathbb{J}=\left(\begin{array}{cccc}
\mu & 0 & 0 & 0 \\
0 & \mu & 0 & 0 \\
0 & 0 & \mu & 0 \\
0 & 0 & 0 & I_{o}
\end{array}\right)
$$

$\mu$ corresponds to linear density and $I_{o}$ to the polar momentum of inertia. We thus propose a simple definition of the spline kinetic energy $T$ :

$$
T=\frac{1}{2} \int_{0}^{L} \frac{d \mathbf{q}^{t}}{d t} \mathbb{J} \frac{d \mathbf{q}}{d t} d s
$$

where ${ }^{t}$ denotes a transpose. Differentiating kinetic energy $T$ with respect to $\mathbf{q}_{\mathbf{i}}$ yields the left term of the Lagrange equations:

$$
\frac{d}{d t} \frac{\partial T}{\partial \dot{\mathbf{q}}_{i}}=\frac{1}{2} \int_{0}^{L} \frac{d}{d t} \frac{\partial \frac{d \mathbf{q}}{d t}^{t} \amalg \frac{d \mathbf{q}}{d t}}{\partial \dot{\mathbf{q}}_{i}} d s
$$

RR n 0123456789 
Replacing $\mathbf{q}$ by the expression given in equation 3 in a similar way as described by Nocent and Rémion [NR01], yields:

$$
\frac{d}{d t} \frac{\partial T}{\partial \dot{\mathbf{q}}_{i}}=\sum_{j=1}^{n} \mathbb{J} \int_{0}^{L}\left(b_{i}(s) b_{j}(s)\right) d s \frac{d^{2} \mathbf{q}_{j}}{d t^{2}}
$$

Considering $\mathbb{J} \int_{0}^{L}\left(b_{i}(s) b_{j}(s)\right) d s$ and $\frac{d^{2} \mathbf{q}_{j}}{d t^{2}}$ as matrices $\mathbb{M}$ and vector $A$ components $\mathbb{M}_{i, j}$ and $\mathbf{A}_{j}$ respectively, this equation yields:

$$
\frac{d}{d t} \frac{\partial T}{\partial \dot{\mathbf{q}}_{\mathbf{i}}}=\sum_{j=1}^{n} \mathbb{M}_{i, j} \mathbf{A}_{j}
$$

Considering all degrees of freedom, this sum or Kinetic term can consequently be written as a matrix-vector product:

To obtain Nocent and Rémion kinetic energy, replace $\mathbb{J}$ by the following matrix: $\left(\begin{array}{ccc}\mu & 0 & 0 \\ 0 & \mu & 0 \\ 0 & 0 & \mu\end{array}\right)$. The use of $\mathbb{J}$ considers twisting inertia.

\subsection{Geometrically Exact energy evaluation}

In this subsection, we propose an expression of the derivatives of strain energies with respect to generalized coordinates. They compose the right term of the Lagrange equations 2;

$$
P^{i}=-\frac{\partial U}{\partial \mathbf{q}_{i}}=-\frac{1}{2} \int_{0}^{L} \frac{\partial\left(\epsilon-\epsilon^{0}\right)^{t} \mathbb{H}\left(\epsilon-\epsilon^{0}\right)}{\partial \mathbf{q}_{i}} d s
$$

they are homogeneous to three generalized forces : stretching force $P_{s}$, twisting force $P_{t}$ and bending force $P_{b}$.

To solve the Lagrange equations, we need to express these generalized forces about position $\mathbf{r}$ and its derivatives $\mathbf{r}^{\prime}, \mathbf{r}^{\prime \prime}, \mathbf{r}^{\prime \prime \prime}$, spline basis functions $b_{i}$ and their derivatives $b_{i}^{\prime}, b_{i}^{\prime \prime}, b_{i}^{\prime \prime \prime}$. This allows us to evaluate the integral terms using classical Riemann sums [PFTV88].

The expressions involving generalized forces are quite complicated, but fast to compute enough. For the sake of clarity and brevity, we will provide them here, but not every detail of the calculation; please see the appendix for more details. We also consider separatly differentiations of strains with respect to position $\mathbf{r}$ and roll $\theta$. Note that $\frac{\partial \mathbf{r}^{(j)}}{\partial \mathbf{r}_{i}}=b_{i}^{j}$ and $\frac{\partial \theta^{(j)}}{\partial \theta_{i}}=b_{i}^{(j)}$ where $b_{i}^{(j)}$ is the $j^{t h}$ derivative of $b_{i}$ with respect to $u$.

Furthermore, we introduce the following variables for compactness of the equations:

$$
\begin{aligned}
& \mathcal{C}=\mathbf{r}^{\prime} \times \mathbf{r}^{\prime \prime} \\
& \mathcal{P}=\frac{\partial \mathbf{r}^{\prime} \times \mathbf{r}^{\prime \prime}}{\partial \mathbf{r}_{i}}
\end{aligned}
$$




$$
\mathcal{T}=\mathcal{C} b_{i}^{\prime \prime \prime}-\mathcal{P} \times \mathbf{r}^{\prime \prime \prime}-2 \tau(\mathcal{C} \times \mathcal{P})
$$

The geometrical twisting $\tau$ will be detailled forward.

We lay stress on the fact again that we consider our object materially linear elastic (small strains) but geometrically exact (large transformations).

Stretching force

In small strains, the stretching strain is defined by $\epsilon_{s}=1-\left\|\mathbf{r}^{\prime}\right\|$. Nocent [NR01] used the large strain assumption,but the difference of accuracy between small strains and large strains is not significative for high rigidities.

The stretching force term $P_{s}^{i}$ yields:

$$
P_{s}^{i}(\mathbf{r})=-\frac{\pi E D^{2}}{4} \int_{0}^{L}\left(1-\frac{\left\|\mathbf{r}_{0}^{\prime}\right\|}{\left\|\mathbf{r}^{\prime}\right\|}\right) \mathbf{r}^{\prime} b_{i}^{\prime} d s
$$

Since stretching strain energy $U_{s}$ does not depend on $\theta$,

$$
P_{s}^{i}(\theta)=0
$$

Twisting force

The twisting comprises two scalar parts: geometrical or Frenet twisting $\tau$ and roll $\theta$. Geometrical twisting is due to the bending of the neutral fiber and is reponsible for bending-twisting coupling, whereas roll corresponds to the rotation of material around the neutral fiber, as described in [BP04]. Chouaeb [Cho04] etablished that twisting is the sum of Frenet twisting and a rotation about the tangent.

The twisting results in the following expression:

$$
\begin{array}{r}
\epsilon_{t}=\frac{d \theta}{d s}+\tau \\
\left\{\begin{array}{l}
\frac{d \theta}{d s}=\frac{\theta^{\prime}}{\left\|\mathbf{r}^{\prime}\right\|} \\
\tau=\frac{\mathbf{r}^{\prime} \times \mathbf{r}^{\prime \prime} \cdot \mathbf{r}^{\prime \prime \prime}}{\left\|\mathbf{r}^{\prime} \times \mathbf{r}^{\prime \prime}\right\|^{2}}=\frac{\mathcal{C} \cdot \mathbf{r}^{\prime \prime \prime}}{\|\mathcal{C}\|^{2}}
\end{array}\right.
\end{array}
$$

This expression is also considered in [GS06].

The geometrical twisting contribution $P_{t}^{i}(\mathbf{r})$ yields:

$$
P_{t}^{i}(\mathbf{r})=-\frac{\pi G D^{4}}{32} \int_{0}^{L}\left(\epsilon_{t}-\epsilon_{t}^{0}\right)\left(\frac{\mathcal{T}}{\|\mathcal{C}\|^{2}}-\frac{\theta^{\prime} b_{i}^{\prime} \mathbf{r}^{\prime}}{\left\|r^{\prime}\right\|^{3}}\right) d s
$$

The roll contribution $P_{t}^{i}(\theta)$ yields:

$$
P_{t}^{i}(\theta)=-\frac{\pi G D^{4}}{64} \int_{0}^{L}\left(\epsilon_{t}-\epsilon_{t}^{0}\right)\left(\frac{b_{i}{ }^{\prime}}{\left\|r^{\prime}\right\|}\right) d s
$$


Bending force

The bending force is a function of the scalar Frenet curvature $k$ which is equal to bending strain $\epsilon_{b}$ :

$$
\epsilon_{b}=k=\frac{\left\|\mathbf{r}^{\prime} \times \mathbf{r}^{\prime \prime}\right\|}{\left\|\mathbf{r}^{\prime}\right\|^{3}}=\frac{\|\mathcal{C}\|}{\left\|\mathbf{r}^{\prime}\right\|^{3}}
$$

Lenoir approximated $k$ by $r^{\prime \prime}$, considering small rotations. In order to have acurate results, we keep the original definition of the curvature in our calculation.

The bending force term $P_{b}^{i}$ yields:

$$
P_{b}^{i}(\mathbf{r})=-\frac{\pi E D^{4}}{64} \int_{0}^{L} \frac{\epsilon_{b}-\epsilon_{b}^{0}}{\left\|r^{\prime}\right\|^{2}}\left(\frac{\mathcal{C} \times \mathcal{P}}{\|\mathcal{C}\|\left\|\mathbf{r}^{\prime}\right\|}-3 k b_{i}^{\prime} r^{\prime}\right) d s
$$

Since bending strain energy $U_{b}$ does not depend on $\theta, P_{b}^{i}(\theta)=0$.

Strain force is the sum of vectors provided by the strain equations in stretching (17), twisting $(20,21)$ and bending $(23)$ and its calculation is expanded in appendix A.

\subsection{Numerical solving of the Lagrange equations}

The Lagrange equations 2 can now be transformed into matrix form,

$$
\mathbb{M} A=F+P
$$

The four sub-systems corresponding to $x, y, z$ and $\theta$ can be solved independently. Since the spline possesses the local control property, the matrix $\mathbb{M}$ is banded with width $2 l-1$, where $l$ is the spline locality.

We solve the system by using a simple LU decomposition at each simulation step. Accelerations are then integrated, at every time step, to determine control point velocities and positions. We use two integration methods: the explicit Runge Kutta method is fast but unstable for high rigidities; the implicit Euler Broyden method described in [HMC01] guarantees numerical stability, but adds damping to the simulation.

We can now simulate an elastic physically-based spline. Adding plasticity to the model only requires a few other considerations, that we describe in the following section.

\section{GEDS in the plastic domain}

In this paper, we may also treat one-dimensional objects as perfectly plastic, and breakable, with a stress-strain curve of the form shown in figure 2 with perfect plasticity. It is possible to simulate real plasticity using a function of $\epsilon$ and $\epsilon_{A}$ which gives the part of the force $\mathcal{F}$ to convert into strain $\epsilon$. In practice, we used ideal plasticity materials: as one can see section 
9.4, our practical results show convincing simulations using ideal plasticity. In addition to that, it is known that such an approximation provides almost exact result for some specific material, such as nylon wires or soft steel. In fact, this curve is quite similar to the curve for nylon wire given by Shuttleworth [SVH99]; this justifies in this paper the use of perfect plasticity.

Stress and strain are not proportional beyond the elastic limits $A_{+}$or $A_{-}$, as shown in Figure 5.

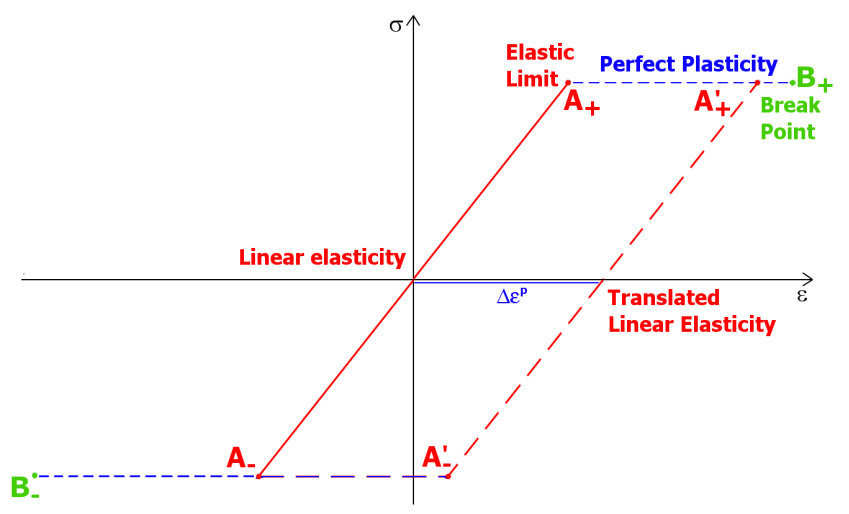

Figure 5: Strain regions that rule one-dimensional objects: linear elasticity, perfect plasticity and break point. $\Delta \varepsilon^{p}$ corresponds to the elastic strain offset induced by a plastic strain.

In perfect plasticity, force cannot exceed the positive yield force $\mathcal{F}_{A_{+}}$or be less than the negative yield stress

mathcal $F_{A_{-}}$; more external works are entirely turned into a plastic strain offset $\Delta \epsilon^{p}$. The elastic region is translated by $\Delta \epsilon^{p}$. When the strain is greater than the break point strain $\epsilon_{B_{+}}$or smaller than $\epsilon_{B_{-}}$, the material irreversibly breaks.

Very little calculation is required to model perfect plasticity or to detect break points. Algorithm 1 can be used separately for each strain scalar component since the strains are all independent. We do not directly use elastic limit stress but we precompute corresponding strains $\epsilon_{A_{+}}$and $\epsilon_{A_{-}}$. We also assume that plastic strain offset $\Delta \epsilon^{p}$ is zero everywhere at the start of the simulation since the material is not damaged yet. At each simulation step, we check if stress $\epsilon^{e}=\epsilon-\epsilon^{0}$ has exceeded the elastic region. If $\epsilon^{e}$ exceeds $\epsilon_{A_{+}}+\Delta \epsilon^{p}$, irreversible strain occurs and the elastic region is translated by their difference $\epsilon_{+}$. So we update $\Delta \epsilon^{p}$ by adding $\epsilon_{+}$.

To deal with plasticity, energy formulation 9 now yields:

$$
U=\frac{1}{2} \int_{0}^{L}\left(\epsilon-\epsilon^{0}-\Delta \epsilon^{p}\right)^{t} \mathbb{H}\left(\epsilon-\epsilon^{0}-\Delta \epsilon^{p}\right) d s
$$




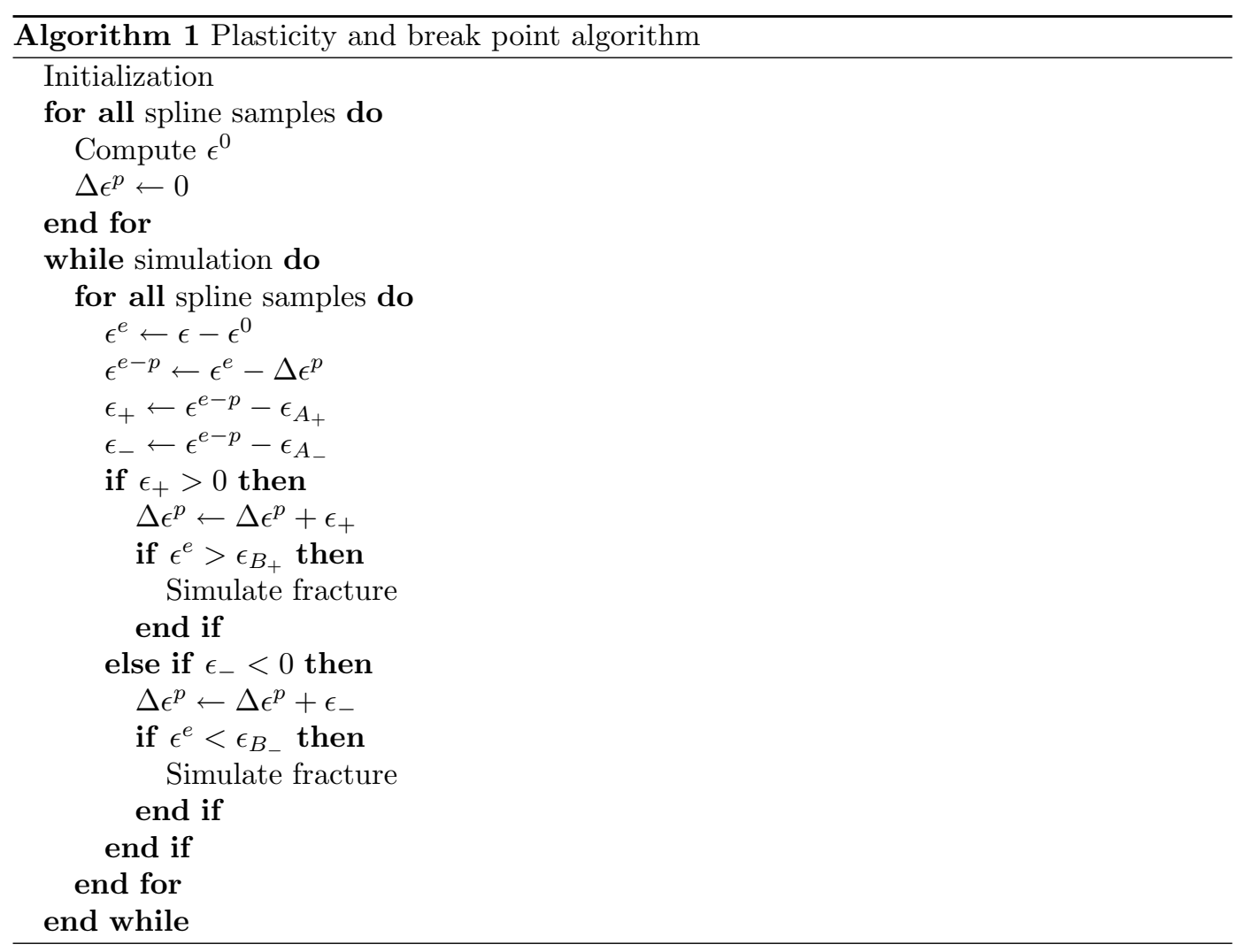


$\epsilon_{A_{+}}$implicitly becomes $\epsilon_{A_{+}^{\prime}}$. When $\epsilon^{e}$ exceeds $\epsilon_{B_{+}}$, the material breaks. The algorithm is the same for negative algebraic values.

\section{$7 \quad$ World interaction}

The spline-based model is continuous, that is, mechanically defined everywhere along the one-dimensional object. An applied force $\mathbf{F}$ on the point $\mathbf{q}$ of the spline provides generalized forces $\mathbf{F}_{i}$. Differentiating the power $W=\mathbf{F q}$ with respect to the generalized coordinate $\mathbf{q}_{i}$, yields the corresponding generalized force $\mathbf{F}_{i}$

$$
\frac{\partial W}{\partial \mathbf{q}_{i}}=\mathbf{F} \frac{\partial \mathbf{q}}{\partial \mathbf{q}_{i}}=\mathbf{F} b_{i}
$$

A force may consequently be applied everywhere, but interacting with the manipulated object remains quite difficult. This is the reason why we use Lagrangian multipliers:they allow us to set the position or the direction of any point of the one-dimensional object. Introducing them into the Lagrange equations 2, we get:

$$
\forall i \in\{1, \ldots, n\},\left\{\begin{array}{l}
\frac{d}{d t}\left(\frac{\partial K}{\partial \dot{\mathbf{q}}_{\mathbf{i}}}\right)=\mathbf{F}_{i}-\frac{\partial T}{\partial \mathbf{q}_{\mathbf{i}}}+\mathbf{L}^{t} \cdot \lambda \\
\phi\left(\mathbf{q}_{\mathbf{i}}, \dot{\mathbf{q}}_{\mathbf{i}}\right)=0
\end{array}\right.
$$

where $L$ is a matrix defined using the different constraints $\phi$ relatively to all degrees of freedom $\left[\mathrm{LGM}^{+} 04\right]$ and $\lambda$ are the Lagrange multipliers which correspond to the force required to maintain the constraints; ${ }^{t}$ still denotes a transpose. The derivated linear system thus yields:

$$
\left(\begin{array}{cc}
\mathbb{M} & \mathbb{L}^{t} \\
\mathbb{L} & 0
\end{array}\right)\left(\begin{array}{c}
A \\
-\lambda
\end{array}\right)=\left(\begin{array}{c}
F+P \\
E
\end{array}\right)
$$

where $\mathbf{E}$ is a vector coding the desirated behaviour of the constraint, position or orientation.

Collision is dealt with a classical penalty method.

\section{Comments about twist control}

\subsection{From mechanical point of view}

A cross-section orientation field is not required to solve mechanics, but only to visualize twisting and apply textures. As a matter of fact, bending and geometrical twisting only depend on control point positions, whereas the roll is not directly considered in the mechanical equations, but its derivative with respect to the spline parameter $u$. A major convenience of our model is that its accuracy does not rely on frames. This allows a real continuity of the one-dimensional object. 


\subsection{From visual point of view}

However, we need to visualize the one-dimensional object to interact with it. A frame that minimizes geometrical twisting has the following major advantage: the aim is to add only a rotation about the tangent of an angle equal to the roll, in the plane of the cross-section. One of the more intuitive frame is due to Frenet. It consists of a unit length tangent $\mathbf{t}$, a principal normal $\mathbf{n}$ and a binormal $\mathbf{b}$. $\mathbf{t}$ is simply the unit length velocity vector $\mathbf{t}=\frac{\mathbf{r}^{\prime}}{\left\|\mathbf{r}^{\prime}\right\|}$. The Frenet frame is convenient because it can be analytically computed at arbitrary point of the curve, but it is undefined wherever the curvature vanishes. Bloomenthal [Blo90] proposed to define an initial frame at the begining of the curve and to propagate it along the curve using small, local rotations. We will use the index $k$ to enumerate frames from the beginning. The rotation matrix $R$ between two frames may be given by the Olinde-Rodrigues formula. Boyer [BP05] gives a convenient expression which does not involve a rotation angle but only the two succeeding units tangents. However, it remains expensive. This is the reason why we use Kenneth Sloan method described by Bloomenthal to propagate the frames:

$$
\left\{\begin{array}{l}
\mathbf{b}_{k}=\mathbf{t}_{k} \times \mathbf{n}_{k-1} \\
\mathbf{n}_{k}=\mathbf{b}_{k} \times \mathbf{t}_{k}
\end{array}\right.
$$

where $\times$ denotes the cross product. To update the frames between two steps $n$ and $n+1$ of simulation, we use the Olinde-Rodrigues formula for its robustness, considering the unit tangents $\mathbf{t}_{\mathbf{0}}^{\mathbf{n}}$ and $\mathbf{t}_{\mathbf{0}}^{\mathbf{n}+\mathbf{1}}$, as described in figure 6 .

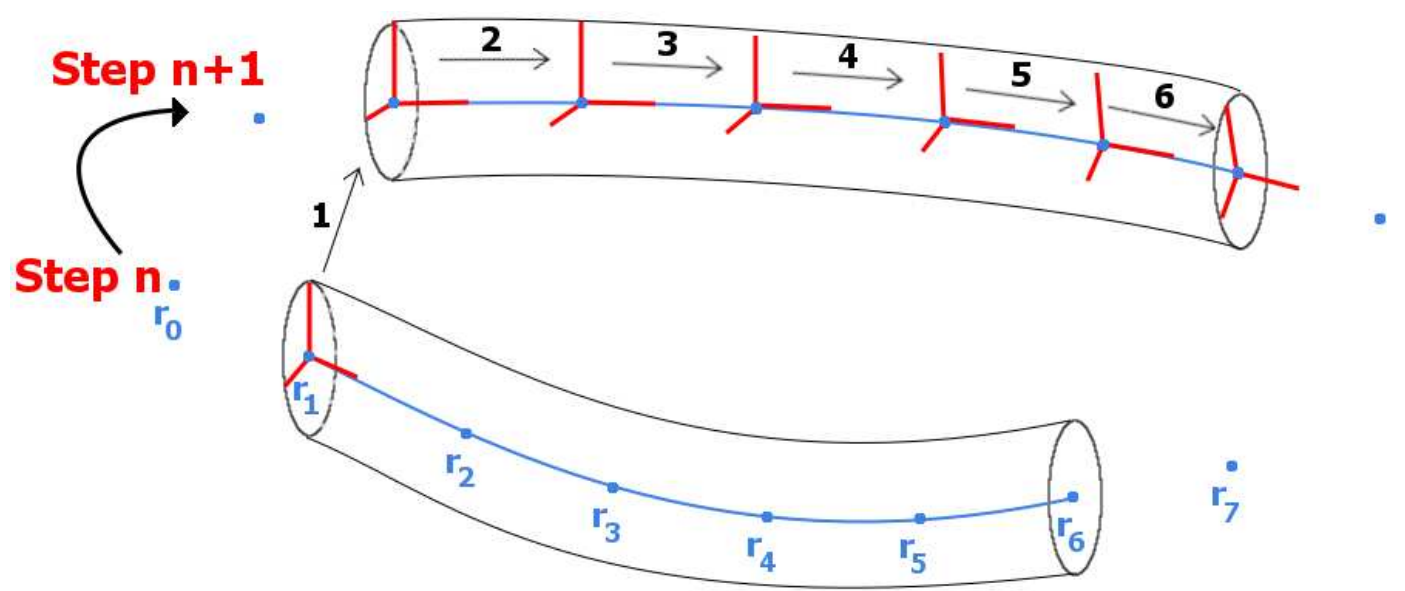

Figure 6: Update of the local frames. 


\section{Implementation and validations}

\subsection{Implementation}

The overall algorithm 2 recalls the required steps to simulate a physically-based spline, including elasticity and plasticity.

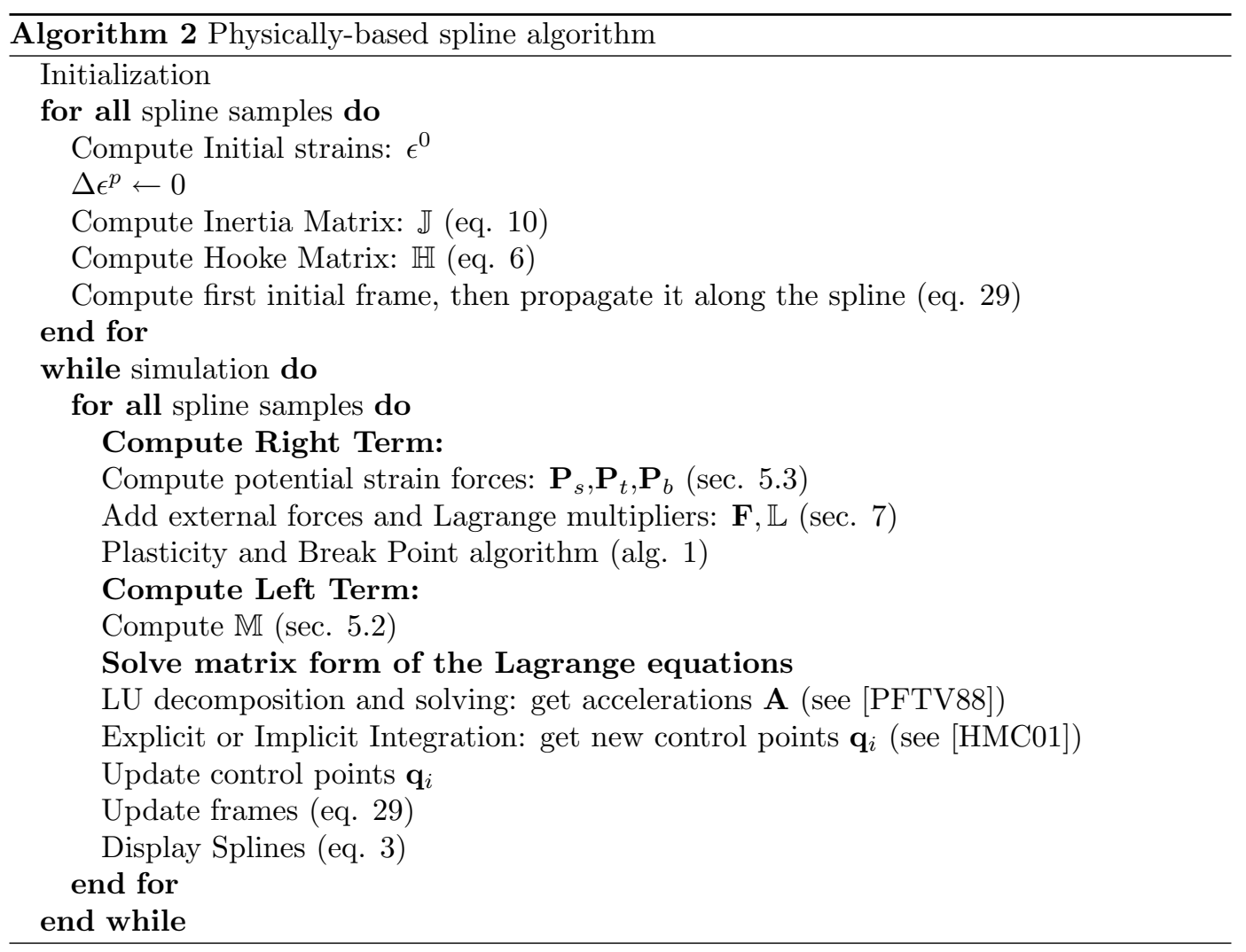

A one-dimensional object is completely defined by a spline specified by an arbitrary number of control points as well as by some physical parameters. These parameters comprise cross-section diameter $D$ and material density $\mu$ as well as two parameters of elasticity (that is, two of the three interrelated constants of Young's modulus, shear modulus and Poisson's ratio). Yield point $A$ and break point $B$ are optional. Strain forces are numerically evaluated, using a classical Riemann sum method with 10 samples per spline. Every point of the spline may be constrained in position and orientation using Lagrange multipliers. We use a red icosahedron as an interactor with the scene. We may attach it to any object via a spring. The spring makes the simulation more robust but introduces oscillations. 
The model described in this paper has been implemented in $\mathrm{C}++$. We performed several tests using a $3 \mathrm{GHz}$ Pentium IV. The following subsections provide some accuracy measurements in static state, as well as performance and realism evaluations in motion state.

\subsection{Classical static states}

To validate our strain formulation, we have performed two classical experiments. The first one is the catenary whose shape is that formed by a perfectly flexible chain suspended by its ends and acted on by gravity. Its equation was obtained by Leibniz, Huygens and Johann Bernoulli in 1691:

$$
y=\frac{h\left(\mathcal{F}_{s}\right)}{S \mu}\left(\operatorname{ch}\left(\frac{S \mu x}{h\left(\mathcal{F}_{s}\right)}\right)-1\right)
$$

where $h\left(\mathcal{F}_{s}\right)$ is the horizontal component of the normal effort $\mathcal{F}_{s}, \mu$ the density and $S$ the area of the cross-section. The shape only relies on stretching and not on bending neither twisting. $h\left(\mathcal{F}_{s}\right)$ can only be numericaly computed with the formulation of the length of the catenary $l$ :

$$
l=\frac{2 h\left(\mathcal{F}_{s}\right)}{S \mu} \operatorname{sh}\left(\frac{S \mu L}{2 h\left(\mathcal{F}_{s}\right)}\right)
$$

$L$ is the distance between the wire ends.

Several configurations have been tested, corresponding to theoretical curves.

The second experiment is the classical problem of deflection of a cantilever beam of linear elastic material, under the action of an external vertical concentrated load at the free end. It was analysed by Beléndez [BNB02]. The beam curvature and deflection only involve bending energy.

The total length $L$ of the beam corresponds to the unknown slope $\phi_{0}$ at the free end of the beam:

$$
L=\sqrt{\frac{E I_{\Delta}}{2 F}} \int_{0}^{\phi_{0}} \frac{d \phi}{\sqrt{\sin \phi_{0}-\sin \phi}}
$$

This equation allows to obtain the slope $\phi_{0}$ at the free end of the beam as a function of the external load $F$. The horizontal and vertical deflection at any point of the neutral axis of the cantilever beam are found as follows:

$$
\begin{gathered}
x=\sqrt{\frac{E I_{\Delta}}{2 F}}\left(\sqrt{\sin \phi_{0}}-\sqrt{\sin \phi_{0}-\sin \phi}\right) \\
y=\sqrt{\frac{E I_{\Delta}}{2 F}} \int_{0}^{\phi} \frac{\sin \phi d \phi}{\sqrt{\sin \phi_{0}-\sin \phi}}
\end{gathered}
$$

The solutions to this problem are elliptic equations, which have no closed form solutions, they have to be numerically solved. Experiments illustrate in figure 11 the fact that theoretical and simulation curves we have computed are close to each other. The solutions to this problem are elliptic equations, which require a numerical solution. Experimental results demonstrate that the simulation curves we have computed are close to the theoretical 
curves, depending on the number of control points per length unit. Convergence of the deflection towards theory relies here on geometrical considerations, not on material ones. The assumption of small strains/large transformations is consequently validated a posteriori.

\subsection{Dynamic simulation}

There are a number of situations that cannot be handled by static simulation.

The motion pendulum is a simple animation that much depends on physical parameters. Moreover, its behaviour corresponds to its material properties, see figures 7 and 2 .
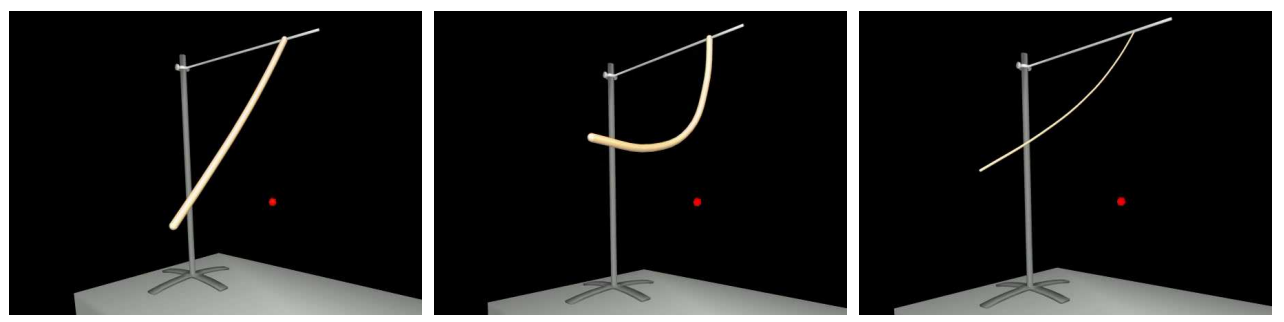

Figure 7: Cantilever beams with several parameters; from top to bottom: $(E=5 e 6 P a$, $D=0.04 m),(E=1 e 6 P a, D=0.04 m),(E=1 e 8 P a, D=0.01 m)$. Varying diameter and Young Modulus induces different behaviours.

The modelization of a spring using an helical version of our model validates bending and twisting initial states as well as energies. It is an interesting example of what can be done with initial states. Springs are treated as purely elastic: they always return to their initial state after being relaxed.

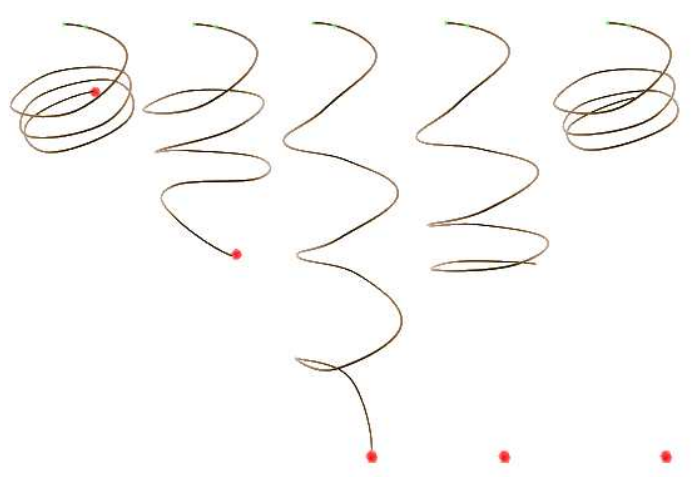

Figure 8: Elongation of spring with 16 control points, $E=1.5 \mathrm{e} 7 \mathrm{~Pa}, \mathrm{D}=1 \mathrm{~cm}$. 


\subsection{Plastic strains and fracture detection}

Plastic deformations can spoil electrical or pneumatic performance in cable laying, whereas in surgery, they enforce suturing quality and are thus welcomed by surgeons. We are able to handle these deformations and determine their magnitude in real-time. When the fracture occurs, its location is indicated by the display of a sphere; here we do not simulate rupture as the object manipulation has failed, but it could be easily done with B-Splines [LGMC05] We illustrate perfect plasticity and fracture in figure 9 .

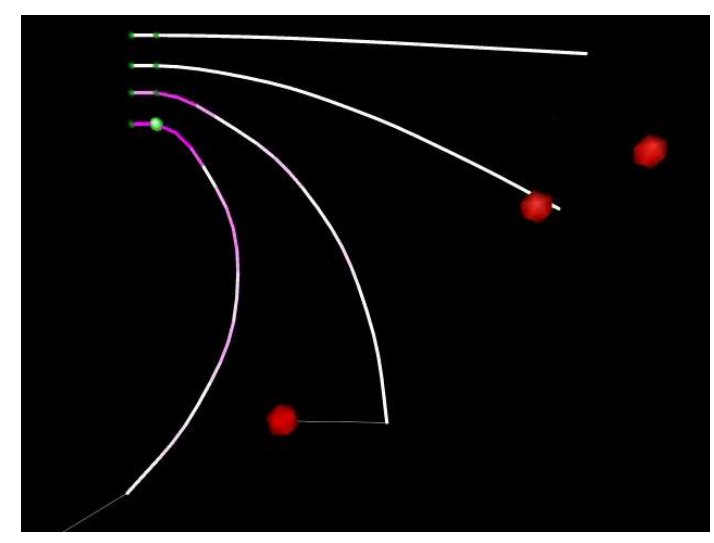

Figure 9: Successive steps from elasticity to plasticity from the companion video. Pink indicates plastic strain intensity and the green sphere tags a break.

\subsection{A practical example of GEDS use in constrained CAD appli- cation}

We illustrate the potential benefit of our model on an application of virtual cable positioning on the inner structure of a car door. A result is shown in figure 1. The purpose is to test compatibility between planned fixing clip positions, and mechanical cable properties. Car engineers still need to build prototypes, since existing solutions are not accurate enough. Our model can prevent them from undergoing this fastidious and inevitable step. The simulation has to address the following properties: elastoplastic stretching, bending, twisting. In this application, fixing clips are mechanically modeled as a set of lagrangian constraints. In our application, we consider simple fixed point constraints; if larger clips would be needed, combination of fixed points, and fixed first derivative constraints would provide satisfactory results. During the interactive manipulation, the cable meets a fixing clip, we create a point constraint. Solving these constraints gives the required forces or multipliers $\lambda$ to maintain the global equilibrium of the cable. If a resulting force overwhelms the fixing clip strength in a determinated direction, the clip fails to keep the cable : the corresponding constraint is deleted. This behavior only requires a simple test to be determinated, which consists in 
the following statement: if the scalar product of the force $\lambda$ with the normal component $N$ of the fixing clip frame is greater than the orientated fixing clip strength, the position constraint is deleted. This test is illustrated in figure 10.

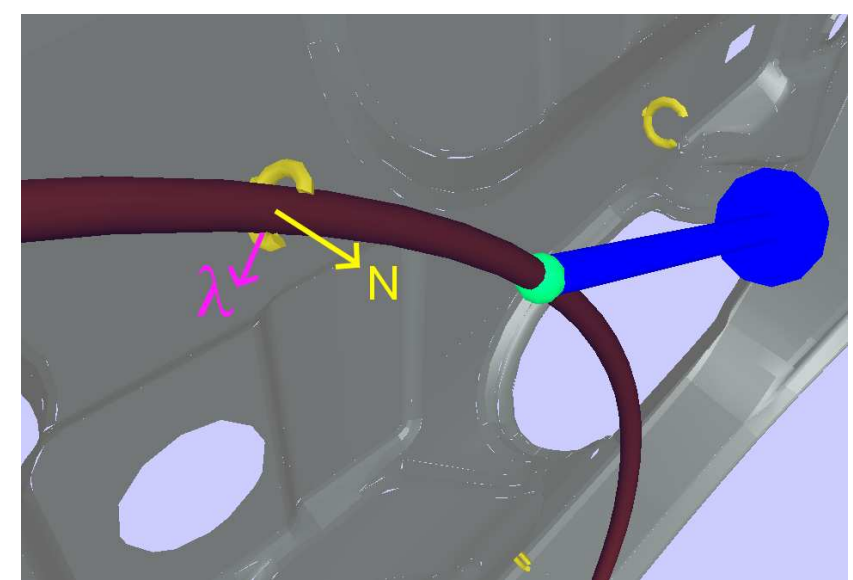

Figure 10: A cable constrained by a fixing clip. Its normal component $N$ and the force $\lambda$ are illustrated by an arrow. The green sphere and its blue arrow correspond to the external force applied to the cable.

Physical parameters and time necessary to compute one step of simulation of $1 \mathrm{~ms}$ in these experiments is provided in table 2. All of these correspond to interactive time and prove the model efficiency.

\section{Conclusion and future work}

Using a background in mechanics consisting of elasticity and plasticity theories, we have proposed a deformable model for one-dimensional objects. Our approach addresses reversible and irreversible deformations, like stretching, twisting and bending, and can even detect fracture. This model provides both accurate mechanical simulation as well as quick calculation. Moreover, we can impose position and orientation everywhere along the object. We can also simulate a wide range of materials in straight or distorted rest states; this is generally at interactive rates, except for very hard stiffness for which the integration method requires excessive computation time for stability. The next step would be to provide a more efficient integration method for our model. Dynamic adaptive repartition of control points would also reduce computation and therefore lower necessary computation time. Finally, adding the capability to handle contact and friction with the environment would improve realism and simulation possibilities. 


\begin{tabular}{|l|c|c|c|c|c|c|c|c|}
\hline Experiment & $n$ & $\begin{array}{c}\ell \\
(\mathrm{m})\end{array}$ & $\begin{array}{c}D \\
(\mathrm{~cm})\end{array}$ & $\begin{array}{c}\mu \\
\left(\mathrm{g} / \mathrm{cm}^{3}\right)\end{array}$ & $\begin{array}{c}E \\
(M P a)\end{array}$ & $\begin{array}{c}\text { PDE } \\
\text { solver }\end{array}$ & $\begin{array}{c}\text { comput. } \\
\text { cost }(\mathrm{ms})\end{array}$ \\
\hline Catenary & 16 & 4.06 & 2 & 6 & 5 & 0.33 & $\mathrm{IE}$ & 0.60 \\
\hline Cantilever & 7 & 1.60 & 4 & 2 & 35 & 0.33 & $\mathrm{IE}$ & 0.57 \\
Cantilever & 7 & 1.60 & 4 & 2 & 70 & 0.33 & $\mathrm{IE}$ & 0.64 \\
Cantilever & 7 & 1.60 & 4 & 2 & 700 & 0.33 & $\mathrm{IE}$ & 0.84 \\
Cantilever & 7 & 1.60 & 4 & 2 & 7000 & 0.33 & $\mathrm{IE}$ & 4.7 \\
\hline Pendulum & 7 & 1.14 & 4 & 4 & 7 & 0.33 & $\mathrm{RK} 4$ & 0.39 \\
Pendulum & 7 & 1.14 & 4 & 4 & 35 & 0.33 & RK4 & 0.41 \\
Pendulum & 7 & 1.14 & 4 & 4 & 700 & 0.33 & RK4 & 0.42 \\
Pendulum & 7 & 1.14 & 1 & 4 & 700 & 0.33 & $\mathrm{IE}$ & 0.94 \\
\hline Buckling & 8 & 3.25 & 4 & 2.4 & 56 & 0.45 & $\mathrm{RK} 4$ & 1.54 \\
Buckling & 8 & 2.00 & 4 & 2.4 & 56 & 0.45 & $\mathrm{RK} 4$ & 1.06 \\
Buckling & 8 & 1.25 & 4 & 2.4 & 56 & 0.45 & RK4 & 0.84 \\
Buckling & 8 & 1.125 & 4 & 2.4 & 56 & 0.45 & RK4 & 0.77 \\
Buckling & 8 & 0.63 & 4 & 2.4 & 56 & 0.45 & RK4 & 0.59 \\
\hline Car door & 18 & 2.00 & 1.08 & 5 & 5000 & 0.33 & IE & 1.30 \\
\hline
\end{tabular}

Table 2: Average calculation time for various material parameters (simulation timestep $1 \mathrm{~ms})$.

\section{A Strain force calculation}

\section{A.1 Stretching force}

The stretching force depends on the square of the stretching strain defined by $\epsilon_{s}=1-\left\|\mathbf{r}^{\prime}\right\|$, that is, $\left(\epsilon_{s}-\epsilon_{s}^{0}\right)^{2}=\left(\left\|\mathbf{r}_{0}^{\prime}\right\|-\left\|\mathbf{r}^{\prime}\right\|\right)^{2}$.

The stretching force term $P_{s}^{i}$ yields:

$$
P_{s}^{i}(\mathbf{r})=-\frac{\partial U_{s}}{\partial \mathbf{r}_{i}}=-\frac{\pi E D^{2}}{8} \int_{0}^{L} \frac{\partial\left(\epsilon_{s}-\epsilon_{s}^{0}\right)^{2}}{\partial \mathbf{r}_{i}} d s
$$

Differentiating $\left(\epsilon_{s}-\epsilon_{s}^{0}\right)^{2}$ with respect to $r_{i}$ yields:

$$
\frac{\partial\left(\epsilon_{s}-\epsilon_{s}^{0}\right)^{2}}{\partial \mathbf{r}_{\mathbf{i}}}=-2\left(\left\|\mathbf{r}_{0}^{\prime}\right\|-\left\|\mathbf{r}^{\prime}\right\|\right) \frac{\partial\left\|\mathbf{r}^{\prime}\right\|}{\partial \mathbf{r}_{i}}
$$

As the derivation of $\left\|\mathbf{r}^{\prime}\right\|$ with respect to $\mathbf{r}_{i}$ is defined by

$$
\frac{\partial\left\|r^{\prime}\right\|}{\partial \mathbf{r}_{i}}=\frac{\mathbf{r}^{\prime} \frac{\partial \mathbf{r}^{\prime}}{\partial \mathbf{r}_{i}}}{\left\|\mathbf{r}^{\prime}\right\|}
$$




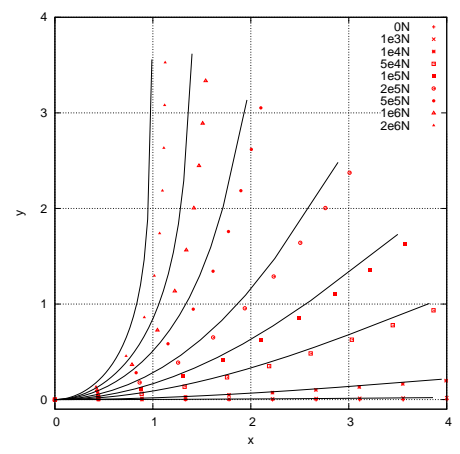

(a) 12 control point spline

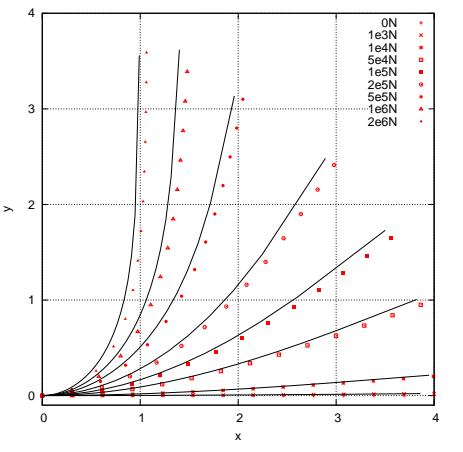

(b) 16 control point spline

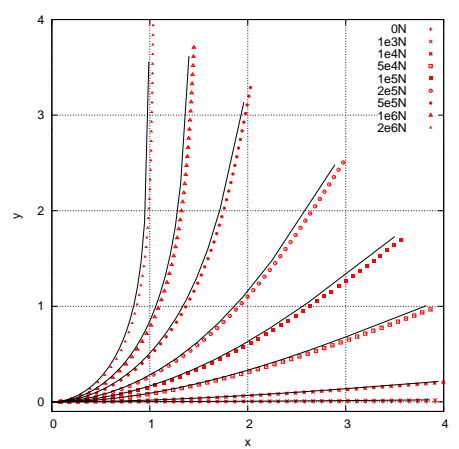

(c) 48 control point spline

Figure 11: Bending validation of our model with deflection of a four meter long cantilever beam, loaded by a force at its end. Dots correspond to simulation results whereas lines are theoritical deflection computed with elliptic equations.

the differentiation results in:

$$
\frac{\partial\left(\epsilon_{s}-\epsilon_{s}^{0}\right)^{2}}{\partial \mathbf{r}_{i}}=2\left(1-\frac{\left\|\mathbf{r}_{0}^{\prime}\right\|}{\left\|\mathbf{r}^{\prime}\right\|}\right) \mathbf{r}^{\prime} b^{\prime}{ }_{i}
$$

Thus, substituting equation 38 in equation 35 results in the following expression for the stretching force:

$$
P_{s}^{i}(\mathbf{r})=-\frac{\pi E D^{2}}{4} \int_{0}^{L}\left(1-\frac{\left\|\mathbf{r}_{0}^{\prime}\right\|}{\left\|\mathbf{r}^{\prime}\right\|}\right) \mathbf{r}^{\prime} b^{\prime}{ }_{i} d s
$$




\section{A.2 Twisting force}

The twisting comprises two scalar parts: geometrical or Frenet twisting $\tau$ and roll $\theta$. Geometrical twisting is due to the bending of the neutral fiber and is reponsible for bendingtwisting coupling, whereas roll corresponds to the rotation of material around the neutral fiber. The twisting results in the following expression:

$$
\begin{array}{r}
\epsilon_{t}=\frac{d \theta}{d s}+\tau \\
\left\{\begin{array}{l}
\frac{d \theta}{d s}=\frac{\theta^{\prime}}{\left\|\mathbf{r}^{\prime}\right\|} \\
\tau=\frac{\mathbf{r}^{\prime} \times \mathbf{r}^{\prime \prime} \cdot \mathbf{r}^{\prime \prime \prime}}{\left\|\mathbf{r}^{\prime} \times \mathbf{r}^{\prime \prime}\right\|^{2}}=\frac{\mathcal{C} \cdot \mathbf{r}^{\prime \prime \prime}}{\|\mathcal{C}\|^{2}}
\end{array}\right.
\end{array}
$$

Frenet twisting is not defined for a straight line, but we assume it is zero in this case.

The geometrical twisting force term $P_{t}^{i}(\mathbf{r})$ yields:

$$
P_{t}^{i}(\mathbf{r})=-\frac{\partial U_{t}}{\partial \mathbf{r}_{i}}=-\frac{\pi G D^{4}}{64} \int_{0}^{L} \frac{\partial\left(\epsilon_{t}-\epsilon_{t}^{0}\right)^{2}}{\partial \mathbf{r}_{i}} d s
$$

Differentiating $\left(\epsilon_{t}-\epsilon_{t}^{0}\right)^{2}$ with respect to $\mathbf{r}_{i}$ yields:

$$
\frac{\partial\left(\epsilon_{t}-\epsilon_{t}^{0}\right)^{2}}{\partial \mathbf{r}_{i}}=2\left(\epsilon_{t}-\epsilon_{t}^{0}\right) \frac{\partial \epsilon_{t}-\epsilon_{t}^{0}}{\partial \mathbf{r}_{i}}
$$

and thus after some calculation:

$$
\frac{\partial\left(\epsilon_{t}-\epsilon_{t}^{0}\right)^{2}}{\partial \mathbf{r}_{i}}=2\left(\epsilon_{t}-\epsilon_{t}^{0}\right)\left(\frac{\mathcal{T}}{\|\mathcal{C}\|^{2}}-\frac{\theta^{\prime} b_{i}^{\prime} \mathbf{r}^{\prime}}{\left\|r^{\prime}\right\|^{3}}\right)
$$

Substituting equation 43 in equation 41, results in the following expression for the geometrical twisting force term:

$$
P_{t}^{i}(\mathbf{r})=-\frac{\pi G D^{4}}{32} \int_{0}^{L}\left(\epsilon_{t}-\epsilon_{t}^{0}\right)\left(\frac{\mathcal{T}}{\|\mathcal{C}\|^{2}}-\frac{\theta^{\prime} b_{i}^{\prime} \mathbf{r}^{\prime}}{\left\|r^{\prime}\right\|^{3}}\right) d s
$$

The roll contribution $P_{t}^{i}(\theta)$ yields:

$$
P_{t}^{i}(\theta)=-\frac{\partial U_{t}}{\partial \theta_{i}}=-\frac{\pi G D^{4}}{64} \int_{0}^{L} \frac{\partial\left(\epsilon_{t}-\epsilon_{t}^{0}\right)^{2}}{\partial \theta_{i}} d s
$$

Differentiating $\left(\epsilon_{t}-\epsilon_{t}^{0}\right)^{2}$ with respect to $\theta_{i}$ yields:

$$
\frac{\partial\left(\epsilon_{t}-\epsilon_{t}^{0}\right)^{2}}{\partial \theta}=2\left(\epsilon_{t}-\epsilon_{t}^{0}\right) \frac{b_{i}{ }^{\prime}}{\left\|r^{\prime}\right\|}
$$

Substituting equation 46 in equation 45, results in the following expression for the roll force term:

$$
P_{t}^{i}(\theta)=-\frac{\pi G D^{4}}{32} \int_{0}^{L}\left(\epsilon_{t}-\epsilon_{t}^{0}\right) \frac{b_{i}{ }^{\prime}}{\left\|r^{\prime}\right\|} d s
$$




\section{A.3 Bending force}

The bending force is a function of the scalar Frenet curvature $k$ which is equal to bending strain $\epsilon_{b}$ :

$$
\epsilon_{b}=k=\frac{\left\|\mathbf{r}^{\prime} \times \mathbf{r}^{\prime \prime}\right\|}{\left\|\mathbf{r}^{\prime}\right\|^{3}}
$$

The bending force term $P_{b}^{i}$ yields:

$$
P_{b}^{i}(\mathbf{r})=-\frac{\partial U_{b}}{\partial \mathbf{r}_{i}}=-\frac{\pi E D^{4}}{128} \int_{0}^{L} \frac{\partial\left(\epsilon_{b}-\epsilon_{b}^{0}\right)^{2}}{\partial \mathbf{r}_{i}} d s
$$

Differentiating $\left(\epsilon_{b}-\epsilon_{b}^{0}\right)^{2}$ with respect to $\mathbf{r}_{i}$ yields:

$$
\frac{\partial\left(\epsilon_{b}-\epsilon_{b}^{0}\right)^{2}}{\partial \mathbf{r}_{i}}=2 \frac{\epsilon_{b}-\epsilon_{b}^{0}}{\left\|r^{\prime}\right\|^{2}}\left(\frac{\mathcal{C} \times \mathcal{P}}{\|\mathcal{C}\|\left\|r^{\prime}\right\|}-3 k b^{\prime}{ }_{i} r^{\prime}\right)
$$

Substituting equation 50 in equation 49, results in the following expression for the bending force term:

$$
P_{b}^{i}(\mathbf{r})=-\frac{\pi E D^{4}}{64} \int_{0}^{L} \frac{\epsilon_{b}-\epsilon_{b}^{0}}{\left\|r^{\prime}\right\|^{2}}\left(\frac{\mathcal{C} \times \mathcal{P}}{\|\mathcal{C}\|\left\|r^{\prime}\right\|}-3 k b^{\prime}{ }_{i} r^{\prime}\right) d s
$$

This expression is nevertheless undefined for $\|\mathcal{C}\|=0$ (i.e. $\mathbf{r}^{\prime \prime}=0$ and $k=0$ ), corresponding to a straight line, but considering a neighborhood of 0 yields a value of the bending force term for the rectilinear state. The magnitude of the tangent vector of the neutral axis must be nonzero; however, $\mathcal{C}$ is zero when the curvature is zero. We may assume that $\frac{\mathcal{C}}{\|\mathcal{C}\|} \sim_{0} \mathbf{1}$. Consequently, the bending force term for the rectilinear state yields:

$$
P_{b}^{i}(\mathbf{r}) \underset{0}{\sim}-\frac{\pi E D^{4}}{64} \epsilon_{b}^{0} \int_{0}^{L} \frac{\mathcal{P} \times \mathbf{1}}{\left\|\mathbf{r}^{\prime}\right\|^{3}} d s
$$

\section{References}

$\left[\mathrm{BAC}^{+}\right.$06] Florence Bertails, Basile Audoly, Marie-Paule Cani, Bernard Querleux, Frédéric Leroy, and Jean-Luc Lévêque. Super-helices for prediciting the dynamics of natural hair. In ACM Transactions on Graphics (Proceedings of the Siggraph Conference), August 2006.

$\left[\mathrm{BKV}^{+} 02\right]$ Pal Benko, Geza Kos, Tamas Varady, Laszlo Andor, and Ralph Martin. Constrained fitting in reverse engineering. Comput. Aided Geom. Des., 19(3):173205,2002 .

[Blo90] Jules Bloomenthal. Calculation of reference frames along a space curve. pages $567-571,1990$. 
[BNB02] Tarsicio Beléndez, Cristian Neipp, and Augusto Beléndez. Large and small deflections of a cantilever beam. European Journal of Physics, 23:371-379, 2002.

[BP04] Frédéric Boyer and Dominique Primault. Finite element of slender beams in finite transformations: a geometrically exact approach. International Journal of Numerical Methods in Engineering, 59:669-702, 2004.

[BP05] Frédéric Boyer and Dominique Primault. Finite element of nonlinear cables : Applications to robotics. Far East Journal of Applied Mathematics, 2005.

[CC09] Eugène Cosserat and François Cosserat. Théorie des objets déformables. Hermann, 1909.

[Cho04] Nadia Chouaeb. Kirchhoff's Problem of Helical Solutions of Uniform Rods and their Stability Properties. PhD thesis, 2004.

[Cou80] Jean Courbon. Théorie des poutres, 1980.

[[Fl] [Flexilution].

[GS06] Mireille Grégoire and Elmar Schmer. Interactive simulation of one-dimensional flexible parts. In Symposium on Solid and Physical Modeling, pages 95-103. ACM, 2006.

[HMC01] Laurent Hilde, Philippe Meseure, and Christophe Chaillou. A fast implicit integration method for solving dynamic equations of movement. In ACM symposium on Virtual reality software and technology, pages 71-76, New York, NY, USA, 2001. ACM Press.

[How01] Larry L. Howell. Compliant Mechanisms. Wiley-Interscience, 2001.

[JL04] Il-Kwon Jeong and Inho Lee. An oriented particle and generalized spring model for fast prototyping deformable objects. Eurographics, 2004.

[JP99] Doug L. James and Dinesh K. Pai. Artdefo : Accurate real time deformable objects. 1999 .

[LCDN06] Julien Lenoir, Stephane Cotin, Christian Duriez, and Paul Neumann. Interactive physically-based simulation of catheter and guidewire. Computer and Graphics, 30(3):417-423, June 2006.

$\left[\mathrm{LGM}^{+} 04\right]$ Julien Lenoir, Laurent Grisoni, Philippe Meseure, Yannick Rmion, and Christophe Chaillou. Smooth constraints for spline variational modeling. In Graphite, pages 58-64, Nanyang Technological University - Singapore, June 15182004. 
[LGMC05] Julien Lenoir, Laurent Grisoni, Philippe Meseure, and Christophe Chaillou. Adaptive resolution of 1d mechanical B-spline. In Graphite, pages 395-403, Dunedin - New Zealand, 29 november-2 december 2005.

[LMGC04] Julien Lenoir, Philippe Meseure, Laurent Grisoni, and Christophe Chaillou. A suture model for surgical simulation. 2nd International Symposium on Medical Simulation (ISMS'04), pages 105-113, june 17-18 2004.

$\left[\mathrm{MDM}^{+} 02\right]$ Matthias Mueller, Julie Dorsey, Leonard McMillan, Robert Jagnow, and Barbara Cutler. Stable real-time deformations. In Symposium on Computer Animation, pages 49-54, San Antonio, Texas, 2002. ACM SIGGRAPH/Eurographics symposium on Computer animation, ACM Press.

[MFLS06] Dominique Michelucci, Sebti Foufou, Loic Lamarque, and Pascal Schreck. Geometric constraints solving: some tracks. In SPM '06: Proceedings of the 2006 ACM symposium on Solid and physical modeling, pages 185-196, New York, NY, USA, 2006. ACM Press.

[NR01] O. Nocent and Y. Rémion. Continuous deformation energy for dynamic material splines subject to finite displacements. In Proceedings of the Eurographic workshop on Computer animation and simulation, pages 88-97, New York, NY, USA, 2001. ACM, Springer-Verlag New York, Inc.

[OBH02] James F. O'Brien, Adam W. Bargteil, and Jessica K. Hodgins. Graphical modeling and animation of ductile fracture. In SIGGRAPH '02: Proceedings of the 29th annual conference on Computer graphics and interactive techniques, pages 291-294, New York, NY, USA, 2002. ACM Press.

[Pai02] D. Pai. STRANDS: Interactive simulation of thin solids using cosserat models. Computer Graphics Forum (Eurographics'02), 21-3, 2002.

[PFTV88] William H. Press, Brian P. Flannery, Saul A. Teukolsky, and William T. Vetterling. Numerical Recipes in C. Cambridge University Press, 1988.

[QT96] Hong Qin and Demetri Terzopoulos. D-NURBS: A physics-based framework for geometric design. In Transactions on Visualization and Computer Graphics, volume 2-1, pages 85-96. IEEE, March 1996.

[RGL05] Stephane Redon, Nico Galoppo, and Ming C. Lin. Adaptive dynamics of articulated bodies. ACM Transactions on Graphics (SIGGRAPH 2005), 24(3), 2005.

[SVH99] Garry N. Shuttleworth, Laurence F. Vaughn, and Hoh B. Hoh. Material properties of ophthalmic sutures after sterilization and disinfection. Journal of Cataract and Refractive Surgery, 25:1270-1274, 1999. 
[TF88] Demetri Terzopoulos and Kurt Fleischer. Modeling inelastic deformation: viscolelasticity, plasticity, fracture. In SIGGRAPH '88: Proceedings of the 15th annual conference on Computer graphics and interactive techniques, pages 269278, New York, NY, USA, 1988. ACM Press.

[TPBF87] D. Terzopoulos, J. Platt, A. Barr, and K. Fleischer. Elastically deformable models. Computer Graphics (Proc. SIGGRAPH'87), 21(4):205-214, 1987.

[WH04] Wakamatsu and Hirai. Static modeling of linear object deformation based on differential geometry. The International Journal of Robotics Research., 23(293311), 2004.

[WSG05] Martin Wicke, Denis Steinemann, and Markus Gross. Efficient animation of point-based thin shells. In Proceedings of Eurographics '05, pages 667-676, 2005 .

[WW92] William Welch and Andrew Witkin. Variational surface modeling. Computer Graphics (Proc. Siggraph), 26(2):157-166, 1992. 
Unité de recherche INRIA Futurs

Parc Club Orsay Université - ZAC des Vignes

4, rue Jacques Monod - 91893 ORSAY Cedex (France)

Unité de recherche INRIA Lorraine : LORIA, Technopôle de Nancy-Brabois - Campus scientifique 615, rue du Jardin Botanique - BP 101 - 54602 Villers-lès-Nancy Cedex (France)

Unité de recherche INRIA Rennes : IRISA, Campus universitaire de Beaulieu - 35042 Rennes Cedex (France)

Unité de recherche INRIA Rhône-Alpes : 655, avenue de l'Europe - 38334 Montbonnot Saint-Ismier (France)

Unité de recherche INRIA Rocquencourt : Domaine de Voluceau - Rocquencourt - BP 105 - 78153 Le Chesnay Cedex (France)

Unité de recherche INRIA Sophia Antipolis : 2004, route des Lucioles - BP 93 - 06902 Sophia Antipolis Cedex (France) 